\title{
Study on Absorption Signal Interference of Gas Concentration Measurement Using Laser Absorption Spectroscopy
}

\author{
Saerom Maeng ${ }^{1,2}$, Sunghyun So ${ }^{1,2}$, Miyeon Yoo ${ }^{1}$, Jungho Hwang ${ }^{2}$ and Changyeop Lee ${ }^{1}$ \\ ${ }^{1}$ Korea Institute of Industrial Technology, Thermochemical Energy System R\&D Group, 31056 Seobuk-gu Cheonan-si \\ Chungcheongnam-do, Republic of Korea \\ ${ }^{2}$ Yeonsei University, Mechanical Engineering, 03722 Seodaemun-gu Seoul, Republic of Korea
}

\begin{abstract}
In order to optimize the combustion condition of the combustion system, it is important to know the information of the physical properties which vary during combustion. Gas concentration and temperature are the major target properties but it is difficult to measure exactly at combustion system. In this paper, a distributed feedback diode laser which wavelength is tunable in accordance with a function generator's output wave is applied to realize the laser absorption spectroscopy measurement. A concentration measuring test for $99 \% \mathrm{CO}_{2}$ gas was performed as basic experiment and major experiments were conducted on separation of interfered absorption signals at $\mathrm{CO}_{2}$ and $\mathrm{CO}$ mixed condition.
\end{abstract}

\section{Introduction}

In order to optimize the combustion condition of a large combustion system such as a powerplant boiler, it is important to obtain the informations about the temperature, the gas concentrations and other properties at various combustion conditions. [1] Generally, a gas analyzer is used to measure the combustion gas concentrations. And a semiconductor sensor, a thermal sensor and an electrochemical sensor are the gas measuring sensors in most gas analyzers. However, these sensors are sensitive to the temperature and humidity changes, sensor replacement is required frequently to secure the reliability.

Almost gas concentration measuring techniques have been limited as sampling type until now, but technical development of the laser absorption spectroscopy enables to measure the internal temperature and the gas concentration of a huge combustion system in real time. [2], [3] It is very important to select a wavelength in this technique because the absorption signals of other species are easily interfered in a wavelength region as shown in Fig. 1. Moreover, there are lots of interference between near absorption signals in a single gas component, it is very difficult to find an independent absorption signal. As shown in Fig. 2, it is good for measuring combustion product gases such as $\mathrm{CO}$ and $\mathrm{CO}_{2}$ at $6250 \sim 6550 \mathrm{~cm}^{-1}$ region, becase there is no $\mathrm{H}_{2} \mathrm{O}$ absorption signal. However, considerable number of signals are interfered by themselves.

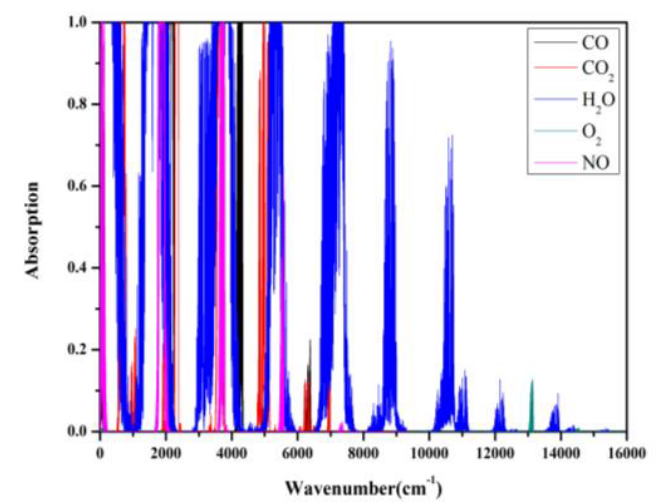

Figure. 1. Absorption spectrum of various chemical species at 296K [4].

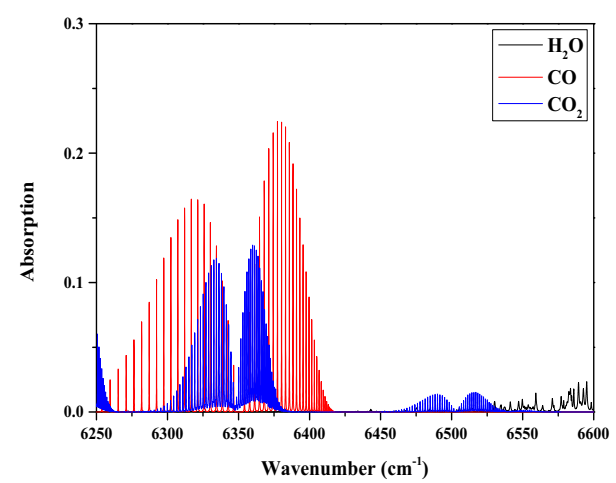

Figure. 2. Absorption spectrum of $\mathrm{CO}, \mathrm{CO}_{2}$ and $\mathrm{H}_{2} \mathrm{O}$ at $296 \mathrm{~K}$ [4]. 
In this paper, a separation method of the interfered absorption signals is suggested to measure the respective gas concentrations of $\mathrm{CO}_{2} \& \mathrm{CO}$ mixture gas and experimental results are discussed with focusing on the measurement error.

\section{Basic principle}

Beer-Lambert law is the basic principle of laser absorption spectroscopy that relates the transmitted intensity $I$ through a medium of length $L$ to the incident intensity $I_{0}$ as

$$
T_{v}=\frac{I}{I_{0}}=\exp \left(-k_{v} \times L\right)
$$

where $T_{v}$ is the transmissivity and $k_{v}$ is the spectral absorption coefficient. $k_{v}$ is expressed as follows.

$$
k_{v}=P X S(T) \varphi_{v}
$$

where $P$ is the pressure, $X$ is the mole fraction of the absorbing species, $S(T)$ the linestrength of the transitions and $\varphi_{v}$ the lineshape function. The $k_{v} L$ is known as the absorbance $\alpha(v)$

$$
\alpha(v)=k_{v} L=-\ln \frac{I}{I_{0}}=P X S(T) \varphi_{v} L
$$

Since the lineshape function $\alpha(v)$ is normalized such that 1 , the integrated absorbance can be expressed as

$$
A=\int \alpha(v) d v=P X S(T) L
$$

Therefore, the mole fraction of absorbing species can be obtained:

$$
X=\frac{A}{P S(T) L}
$$

\section{Experimental setup}

Fig. 3 shows the optical experimental system layout to measure the gas concentration using laser absorption spectroscopy. We used a distributed feedback laser(DFB laser) as a light source and wavenumber of the light is about $6371 \mathrm{~cm}^{-1}$ where the $\mathrm{CO}_{2}$ and $\mathrm{CO}$ gas absorption signals including overlapping signals are variously distributed. And the wavelength was modulated slightly using a function generator. And an aluminum oxide $\left(\mathrm{Al}_{2} \mathrm{O}_{3}\right)$ tube was used to trap the target gas. $\mathrm{CO}_{2}$ \& $\mathrm{CO}$ mixture gas was used to evaluate the utility of proposed signal separation method.
The modulated laser light was divided by two direction through a $1 \times 2$ coupler, the first one employed an incident light passing a solid etalon. The solid etalon was used for wavelength conversion. The second light was changed into parallel light through the collimating lens(CL1), passed the gas medium inside the aluminum oxide tube and then arrived at the photo detector(PD2). Then optical absorption signals were obtained and calculated by analysis algorithms.

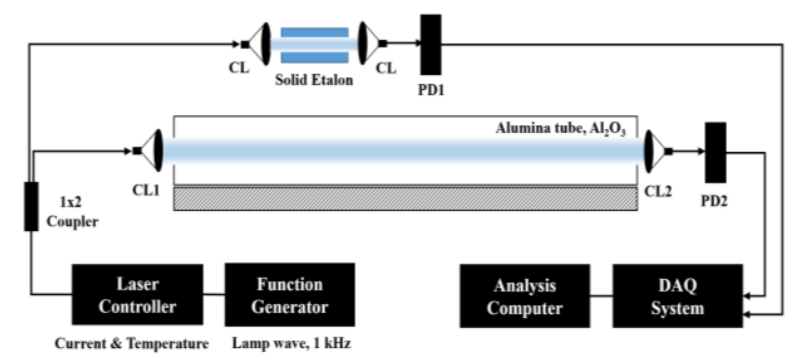

Figure. 3. Optical measurement system layout (CL: collimateng lens, PD: photo detector)

\section{Results}

Fig. 4 shows the absorption signals of the $\mathrm{CO}_{2}$ gas $99 \%$ and $\mathrm{CO}_{2} 85 \%$ \& $\mathrm{CO} 15 \%$ mixture gas in the same wavelength region. When single gas $\left(\mathrm{CO}_{2} 99 \%\right)$ is distributed in the tube, Only one absorption signal was observed. And two absorption signals were observed at mixed gas $\left(\mathrm{CO}_{2} 85 \%\right.$ \& $\left.\mathrm{CO} 15 \%\right)$ condition. As shown in Fig.4 (b) graph, it is difficult to decide the absorption area that is essential for calculating concentration because of $\mathrm{CO}_{2}$ and $\mathrm{CO}$ signal overlapping.

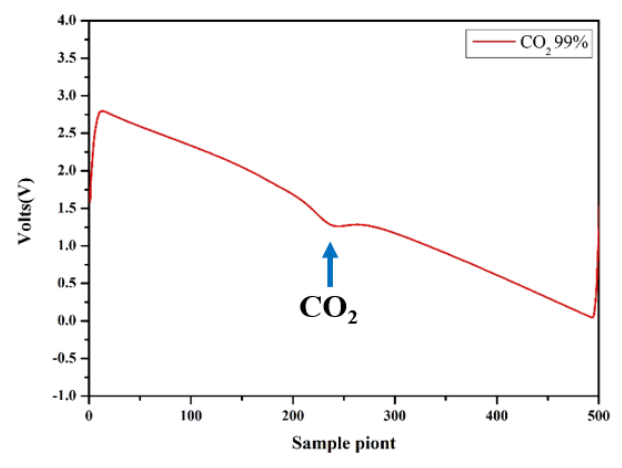

(a) Absorption signal $\mathrm{CO}_{2}$ gas

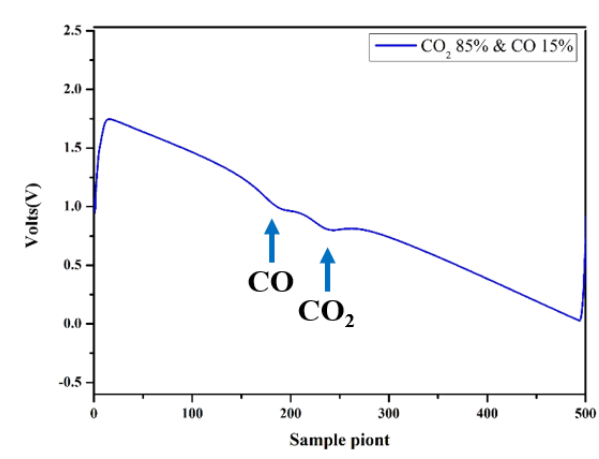

(b) Absorption signal $\mathrm{CO}_{2} \& \mathrm{CO}$ mixed gas 


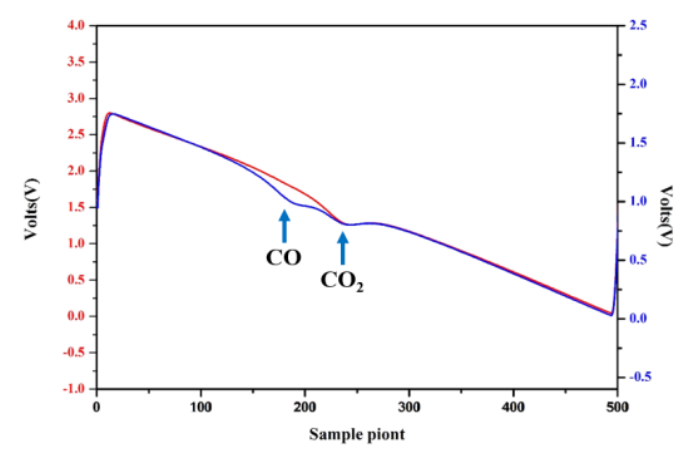

(c) Comparison of absorption signals

Figure. 4. Absorption signal measurement data of $\mathrm{CO}_{2}$ and $\mathrm{CO}_{2}$ \& $\mathrm{CO}$ gas

However the single absorption lineshape of $\mathrm{CO}_{2}$ gas is symmetric as shown in Fig.5 (a). Then, respective lineshapes can be inferred from the interfering signal as considering the symmetric characteristic of absorption signal. It is possible only when signals are not totally overlapped, in other words, each signal's full maximum height point and more than half absorption lineshape should be exposed.

Figure 5 (b) Graph shows the inferred absorption lineshapes, the dotted lineshapes are inferred. Table 1 shows the analysis results of absorption signals. Precise concentration analysis had been achieved in case 1 , when the absorption signal was isolated alone. Case 2 shows the result of each inferred absorption lineshapes which are inferred by above mentioned manner. Measurement gas concentrations of $\mathrm{CO}_{2}$ and $\mathrm{CO}$ were calculated by $93.76 \%$ and $18.12 \%$ each, however, the real gas concentrations were $85 \%$ and $15 \%$. The calculate values were bigger than real values. It results from the problem of inference method. When the overlapping occurs between two absorption signals, it is expected that overlapped parts of the combined absorption signal contribute to make higher full maximum heights of the signal. Because respective absorption lineshapes were inferred from already higher height signals, absorption area values were calculated larger as well and they induced bigger predicted concentration values. The problem was also found to check the absorbance area. The area sum of inferred absorption lineshapes was $15.07 \%$ bigger than real absorbance area of combined absorption signal.

Case 3 of Table 1 shows the result of compensating analysis method. In this case, inferred absorption lineshapes were used to find the area ratio information only between $\mathrm{CO}_{2}$ and $\mathrm{CO}$ gas absorption signals. And quantitative values were obtained from integral calculus of combined absorption signal. As the area ratio of two inferred absorption lineshapes were 0.56 versus 0.44 , real absorbance was divided by that ratio. And calculated gas concentrations of $\mathrm{CO}_{2}$ and $\mathrm{CO}$ using this manner were $81.12 \%$ and $15.69 \%$ respectively. Error rates of case 3 were reduced considerably than those of case 2 .

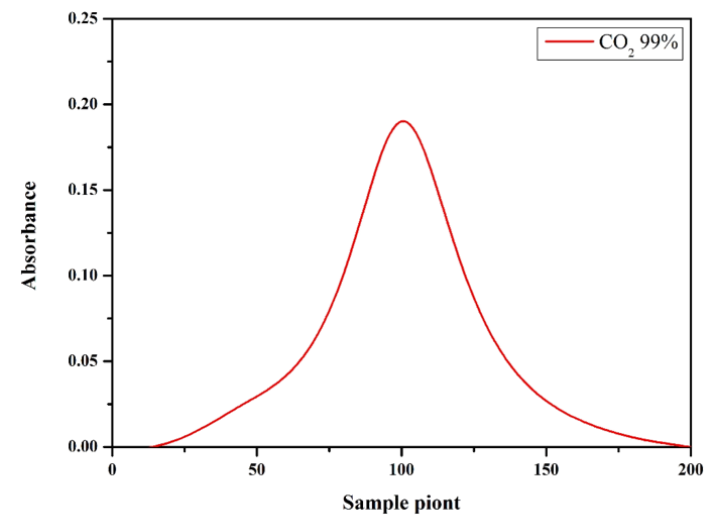

(a) Absorption lineshape of $\mathrm{CO}_{2}$ gas

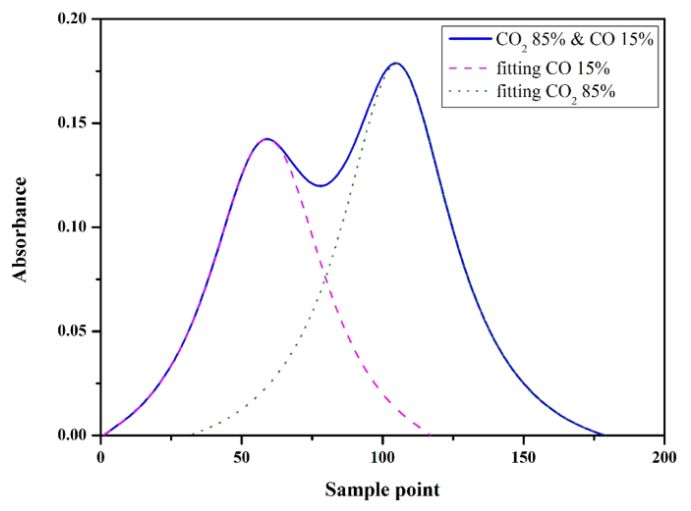

(b) Absorption lineshape of $\mathrm{CO}_{2}$ \& $\mathrm{CO}$ mixed gas

Fig. 5. Absorbance measurements

Table 1. Setting Word's margins.

(a) Case 1

\begin{tabular}{|c|c|}
\hline Gas species & $\mathbf{C O}_{\mathbf{2}} \mathbf{( 9 9 \% )}$ \\
\hline Measured concentration & $99.04 \%$ \\
\hline Error rate & $0.04 \%$ \\
\hline
\end{tabular}

(b) Case 2

\begin{tabular}{|c|c|c|}
\hline Gas species & $\mathbf{C O}(\mathbf{1 5 \%})$ & $\mathbf{C O}_{\mathbf{2}}(\mathbf{8 5} \%)$ \\
\hline Measured concentration & $18.14 \%$ & $93.76 \%$ \\
\hline Error rate & $20.9 \%$ & $10.3 \%$ \\
\hline
\end{tabular}

(c) Case 3

\begin{tabular}{|c|c|c|}
\hline Gas species & $\mathbf{C O}(\mathbf{1 5 \%})$ & $\mathbf{C O}_{\mathbf{2}} \mathbf{( 9 9 \% )}$ \\
\hline Measured concentration & $15.69 \%$ & $81.124 \%$ \\
\hline Error rate & $4.6 \%$ & $4.56 \%$ \\
\hline
\end{tabular}




\section{Conclusion}

The optical absorptions of $\mathrm{CO}_{2}$ and $\mathrm{CO}$ gas are occurred in the infrared region, and many of them are overlapped signals that precise informations are not revealed. In this paper, lineshapes were inferred from the interfering signal as considering the symmetric characteristic of absorption signal at partially overlapped condition. As a results, respective gas concentrations were successfully calculated using absorbance area ratio of inferred lineshape and real integral area value of combined absorption signal. The possibility that the interfered absorption signals can be used to analyze the gas concentration and temperature has been confirmed through this study. It will useful to analyze the physical properties at high temperature and multi-species mixed condition.

\section{Acknowledgement}

This work was supported by the Korea Institute of Energy Technology Evaluation and Planning(KETEP) and the Ministry of Trade, Industry \& Energy(MOTIE) of the Republic of Korea (No. 20152010103630).

\section{References}

1. M. A. Allen, E. R. Furlong, R. K. Hanson, Applied Combustion Diagnostics, edited by K. KohseHoinghaus and J.B Jeffries, Taylor and Francis, New York (2002)

2. R. M. Mihalcea, M. E. Webber, D. S. Baer, R. K. Hanson, G. S. Feller, a W. B. Chapman, Appl. Phys. B, 67 (1998)

3. A. Farooq, H. Li, Jay B. Jeffries, and R. K. Hanson, 43rd AIAA/ASME/SAE/ASEE Joint Propulsion Conference \& Exhibit Cincinnate, AIAA

4. HITRAN DATABASE infermation on http://hitran.iao.ru/ 